\title{
Measuring asymmetric temporal interdependencies in simulated and biological networks
}

\author{
Rhonda Dzakpasu \\ Department of Physics University of Michigan, Ann Arbor, Michigan 48109 \\ Kinjal Patel, Natallia Robinson, and Melissa A. Harrington \\ Department of Biological Sciences Delaware State University, Dover, Delaware 19901 \\ Michał Żochowski \\ Department of Physics and Biophysics Research Division University of Michigan, \\ Ann Arbor, Michigan 48109
}

(Received 23 August 2006; accepted 2 November 2006; published online 7 December 2006)

\begin{abstract}
We use a newly developed metric to characterize asymmetric temporal interdependencies in networks of coupled dynamical elements. We studied the formation of temporal ordering in a system of coupled Rössler oscillators for different connectivity ratios and network topologies and also applied the metric to investigate the functional structure of a biological network (cerebral ganglia of Helix snail). In the former example we show how the local ordering evolves to the global one as a function of structural parameters of the network, while in the latter we show spontaneous emergence of functional interdependence between two groups of electrodes. (C) 2006 American Institute of Physics. [DOI: 10.1063/1.2401130]
\end{abstract}

It is thought that patterns of synchronous activity underlie information processing in neural systems. The formed temporal patterns may include randomly ordered coincidence of activations of neuronal populations or their functionally relevant temporal ordering. We have developed a metric that is able to detect temporal ordering between the elements within a network. The metric is based on the asymmetric analysis of stability of lead/lag patterns between dynamical units forming the network. We show that calculation of those asymmetric temporal interdependencies between the elements aids in the identification of the pattern formation in the network as a whole, which in turn may shed light on the functional network structure. Specifically, we show applicability of the metric in the study of the emergence of ordering in the network of nonidentical Rössler oscillators having sparse directional diffusive coupling and also in biological networks of neurons populating cerebral ganglia of the brown garden snail. We show how emergence of the global ordering in Rössler network depends on the structural parameters of the graph, and also identify elements of functional structure of the cerebral ganglion network.

\section{INTRODUCTION}

Spatio-temporal pattern formation has been studied extensively in various physical and natural systems. The brain is an example of a natural system where the evolving temporal correlations between the neurons are thought to, on one hand, underlie information processing, but at the same time can be a signature of serious pathology; e.g., an epileptic seizure. ${ }^{1,2}$ Thus, the detection and characterization of temporal interdependencies between individual neurons or neuronal populations may lead to a better understanding of the role that time plays in network dynamics. From a biological and cognitive standpoint, Von der Malsburg and others ${ }^{3-6}$ have hypothesized that synchrony plays a crucial role during information processing because it allows for functional binding of neural ensembles coding the same feature. Along those lines, Laurent ${ }^{7}$ has shown that abolition of oscillatory response in the olfactory system of honeybees may impede discrimination of similar odorants.

At the same time, $\mathrm{Bi}$ and $\mathrm{Poo}^{8}$ established experimentally that potentiation of synapses between neurons (the neurophysiologic process underlying learning) is directly linked to the causal relationship between their firing patterns. Thus, it is clear that not only mere coincidence of activations between cells is important for neural systems, but the causal locking between them as well. Similarly, in the case of neural populations causal locking may underlie the direction of information transfer between different neuronal ensembles that leads to cognitive processing.

On the other hand, detection of causal interdependencies between neural populations during epileptic seizures is crucial for understanding the dynamics of the seizure progression and for the identification of its focus ${ }^{9}$ that could allow for development of better treatment options.

Since the brain changes states rapidly, it becomes pertinent to develop adaptive metrics that can monitor instantaneous causal interdependencies between neurons and to distinguish them from mere temporal coincidence, when neurons fire more or less at the same time but actual temporal ordering is not established. Any method designed for this purpose must be statistical, based on information obtained from multiple events but at the same time be able to detect dynamical changes in the system.

Multiple techniques have been developed to capture formation of such temporal interdependencies. Many of these operate in a pairwise manner on the inner product of two 
signals (usually time series or frequency spectrum) (see Ref. 10 for a brief review). Such methods include cross-intensity functions, ${ }^{11}$ product densities, cumulant densities, cumulant spectra, ${ }^{12}$ cross-correlation, ${ }^{13}$ joint peristimulus time histogram, ${ }^{14}$ and coherence. ${ }^{15}$ Others operate on the entire phase space of recorded interactions. These include dimensional reduction methods, such as principle component analysis and multiple discriminant analysis, ${ }^{16}$ optimal linear estimation, ${ }^{17}$ Bayes methods, ${ }^{18}$ maximum likelihood methods, ${ }^{19}$ and a novel method of functional holography that reconstructs the functional space of interactions. ${ }^{20}$

Previously, we developed a metric (causal entropies) based on the analysis of the distribution of relative interevent timings between two coupled oscillators that monitors the formation of a phase/lag synchronized state. ${ }^{21}$ We have also shown that the metric has advantages over correlationbased techniques as it can detect asymmetry in the temporal relations between the oscillators. ${ }^{22}$ Here we extend the use of this measure to analyze temporal interdependencies in networks of interacting dynamical elements. The measure is used to detect temporal ordering between pairs of individual oscillators as well as on the scale of the whole network. We show that detection of phase/time lag interdependencies between the elements of the network may provide information about the lead-lag pattern observed in the network and help in characterization of its functional structure.

We applied the measure to a network of interacting, nonidentical Rössler oscillators and also to multielectrode array recordings from a neuronal network formed in the cerebral ganglia of Helix snails. This is an in vitro preparation with the sensory epithelia intact. We show that during one phase of the experiment, there is a stable lead/lag pattern between two groups of electrodes that allows us to infer a functional relationship.

Recently, novel methods have been proposed to assess information transfer based on analysis of phase synchrony between coupled systems ${ }^{23}$ and the introduction of the chaotic channel of interaction formed between connected chaotic systems. ${ }^{24}$ The former analyzes the conditional Poincaré maps that allows for assessment of phase synchrony without direct phase measurement, whereas the latter allows for estimate of the amount of information transferred between the transmitter and the receiver element. Also, comparison of different metrics that measure coupling direction can be found in Ref. 25.

There are also other methods that use inter-event timings to assess information transfer between two coupled oscillators. ${ }^{26,27}$ For example, in Ref. 26 the inter-event timings are used to directly calculate the mutual information from two coupled Hindmarsh-Rose neurons. Our algorithm is similar to that of Ref. 27, but instead of quantifying the lag values we investigate lag stability, which allows us to generalize our results independent of the amount of lag.

\section{MEASUREMENT OF DIRECTIONALITY OF TEMPORAL INTERDEPENDENCE FOR THE NETWORK OF COUPLED OSCILLATORS}

To detect temporal interdependencies in a network of coupled oscillators we apply a measure based on the dy-

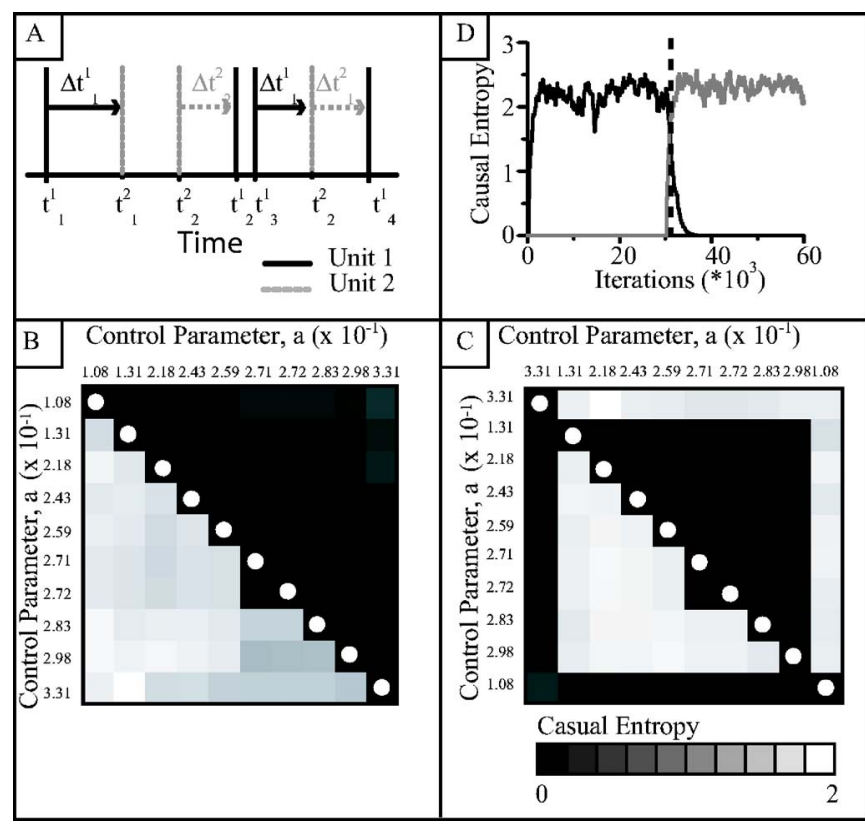

FIG. 1. (A) Definition of inter-event times used for calculation of asymmetric interactions. (B),(C) Spatial maps of the CE array for a network of 10 fully connected Rössler oscillators. (B) The array is ordered with respect to the parameter values $a_{i}$. (C) The parameters of the oscillator with the lowest and highest parameter values are exchanged during the simulation. The $\mathrm{CE}$ array is calculated for 30000 iterations after the switch. The change in the phase lag distribution within the network can be observed. (D) Evolution of $\mathrm{CE}_{12}$ and $\mathrm{CE}_{21}$ as a function of time; the values reverse rapidly after the oscillator's parameter is switched.

namic evaluation of temporal locking between the elements in the network. This measure monitors the correlations in inter-event intervals (IEIs) between the interconnected oscillators. ${ }^{21}$ In the case of the Rössler oscillator, an event is defined to be the time when the trajectory of the oscillator crosses a defined Poincaré section. To apply the measure to neurobiological data, the event could be the spike time or the timing of any other characteristic event in biology in general. The underlying idea of the measure is that the variability in the IEIs between oscillators that are temporally locked will be significantly smaller than the IEIs between independent oscillators. Thus, this analysis is limited to the case of a system composed of two elements in which at least one of them is generating an aperiodic time series of events. In the case in which both of the oscillators are periodic, the direction of the locking cannot be determined. The aperiodicity could be due in general to intrinsic fluctuations of the system or its chaotic nature. For the case of a chaotic system the aperiodicity in the entropic difference could be in principle linked to the existence of positive Liapunov exponents transversal to the synchronous solution of the system.

Individual distributions are formed for each oscillator using the relative durations of the IEI of the oscillator with respect to the others in the network. Specifically, the IEI $\Delta t_{m}^{i j}$ of the $j$ th unit with respect to the $i$ th unit is calculated as a time difference between the inter-event timing of the $j$ th unit with respect to the last event of the $i$ th unit, and conversely the inter-event interval $\Delta t_{m}^{j i}$ of the $i$ th unit is calculated as a time difference between the event of unit $i$ with respect to the timing of the last event taking place on the $j$ th unit [Fig. 
1(A)]. Thus, if $t_{1}^{i}$ and $t_{2}^{i}$ are the consecutive event timings of the $i$ th unit (with $t_{1}^{i}<t_{2}^{i}$ ), $t_{1}^{j}$ and $t_{2}^{j}$ are the event timings of the $j$ th unit (with $t_{1}^{j}<t_{2}^{j}$ ), and if $t_{1}^{i}>t_{1}^{j}>t_{2}^{i}>t_{2}^{j}$, then one distribution (calculated from events occurring on $j$ th element) will be updated with only one inter-event interval $\left(\Delta t^{j i}=t_{2}^{i}-t_{1}^{j}\right)$, whereas the second distribution (calculated with respect to events of element $i)$ will be updated twice with $\left(\Delta t^{i j}=t_{1}^{j}\right.$ $\left.-t_{1}^{i}\right)$ and $\left(\Delta t^{i j}=t_{2}^{j}-t_{2}^{i}\right)$. The distributions defined in this manner are complementary to each other, providing complete information about possibly asymmetric timing interdependencies between the coupled oscillators.

The distributions are updated as new events are generated to allow dynamical monitoring of the change in the relative event timings of the coupled oscillators. The bin of the appropriate distribution within which the latest IEI falls is increased by a fixed $\Delta P$. After every update, the appropriate distributions are renormalized. Thus,

$$
\begin{aligned}
& \Delta P_{I}(t+1) \\
& \quad=\left\{\begin{array}{cl}
\frac{\Delta P-\Delta P P_{I}(t)}{1+\Delta P} & \text { if the bin } P_{I} \text { was updated, } \\
\frac{-\Delta P P_{I}(t)}{1+\Delta P} & \text { if the bin } P_{I} \text { was not updated. }
\end{array}\right.
\end{aligned}
$$

Lastly, the entropy, $S=-\Sigma_{I} P_{I} \log _{10} P_{I}$, is calculated. Here the entropy function is not used in the standard information theoretic sense but to estimate the stability of the IEI within the created distribution. If the pairwise comparison of those entropies yields a significant difference in their values, this is indicative of the existence of asymmetry in their functional coupling.

The parameter $\Delta P$ is a free parameter of the measure that determines how much the distributions depend on the newest events: if $\Delta P$ is small, a single event will not significantly change the distributions, whereas when $\Delta P$ is large the distribution is heavily skewed towards the newest event.

We refer to the measure as that of a causal entropy (CE) since we use changes in distribution entropy to determine the evolution in the causal interdependence between the two oscillators.

As a result of the pairwise comparison, an $N \times N$ causal entropy matrix is formed for all $N$ oscillators in the network, with elements $(i, j)$ and $(j, i)$ providing information about the degree of relative locking between two elements in the network. Often it is useful, however, to depict the existing relations in equivalent form as the linear combination of the entropic differences; namely, $\mathrm{CED}_{i j}=S_{i j}-S_{j i}$ and $\mathrm{CES}_{i j}=S_{i j}$ $+S_{j i}$. The parameter $\mathrm{CED}_{i j}$ measures effectively the existence of the asymmetry in the interaction and its direction. The $\mathrm{CES}_{i j}$, on the other hand, represents joint entropy of the interaction between the two units. In the ideal situation, the $\left(S_{i j}, S_{j i}\right)$ and/or $\mathrm{CED}_{i j}$ and $\mathrm{CES}_{i j}$ pairs can take three distinctly different values:

- $S_{i j} \simeq S_{j i} \simeq 0$; equivalently $\mathrm{CED}_{i j}, \mathrm{CES}_{i j} \simeq 0$ usually implies complete synchronization of both oscillators (for example when both oscillators are identical, i.e., having the same parameters), or the fact that both processes are periodic. In this case, temporal interdependence (if it exists) cannot be determined solely on lag variability.

- $S_{i j} \simeq 0$ and $S_{j i} \neq 0$ (or vice versa); equivalently $\mathrm{CED}_{i j}, \mathrm{CES}_{i j} \neq 0$ implies asymmetric distributions. The two signals exhibit temporal locking and the lead/lag direction can be inferred from the sign of the $\mathrm{CED}_{i j}$.

- $S_{i j} \simeq S_{j i} \neq 0$; equivalently $\mathrm{CED}_{i j} \simeq 0, \mathrm{CES}_{i j} \neq 0$ indicates that both units are aperiodic and there is no detectable ordering between the two signals. This could imply that the signals are completely independent, or coincident but with randomly changing lead/lag pattern. In both cases there is no detectable direction of information flow.

Thus, the measure can identify the existence of directionality in information flow defined as systematic lead/lag pattern that could be indicative of functional coupling between the elements of the network. This lead/lag pattern could be a result of the gross anatomical structure of the network, or (when such is not present) dynamically changing, relative properties of coupled network elements (i.e., differences in intrinsic frequencies in the case of Rössler oscillators, or dynamically changing input in the case of biological neurons). The latter one in principle could allow for dynamical changes in the information flow in the network dependent on the computed content.

\section{MEASUREMENT OF TEMPORAL ORDERING IN A SIMULATED NETWORK}

It is well established that nonidentical, nonlinear oscillators may undergo phase or lag synchronization. ${ }^{28-31}$ The detection of relative properties of the phase-synchronized state of the coupled oscillators can be used to shed light on the dynamical evolution of the network itself. We use the CE metric to detect global temporal ordering in a pair of coupled, nonlinear oscillators to a system of coupled Rössler oscillators. The Rössler network is defined as follows:

$$
\begin{aligned}
& \dot{x}_{i}=-\left(z_{i}+y_{i}\right), \\
& \dot{y}_{i}=x_{i}+a_{i} y_{i}+\frac{\alpha}{M} \sum_{j \in C_{i}}^{M}\left(y_{j}-y_{i}\right), \\
& \dot{z}_{i}=b+\left(x_{i}-c\right) z_{i},
\end{aligned}
$$

where the subscript denotes the oscillator number; $a_{i}, b$ $=0.2$, and $c=10.0$ are the parameters of the oscillators; $\alpha$ $=0.4$ denotes the coupling strength; and $N$ is the number of oscillators. The values of parameters $a_{i} \in(0.1,0.35)$ are different for every oscillator and are generated at random from a uniform distribution. This parameter controls the type of the attractor and modifies the frequency of the oscillator. ${ }^{31}$ Parameter $M$ defines number of connections per element, so that $M \leq N-1$ and is dependent on the connectivity in the network; for a one-dimensional network, $M$ is given in terms of radius of connectivity $R$, so that $M=2 R ; C_{i}$ represents the set of all existing connections of $i$ th oscillator. The connections are directional; i.e., existence of a connection from $i$ to $j$ does not imply the existence of the reverse connection. The event is defined to be the time of crossing of the oscillator trajectory with the Poincaré section $z=1$. The equations are 
integrated using the Runge-Kutta algorithm with a step size of $h=0.01$.

We have shown earlier that the pairwise calculation of the causal entropies provides asymmetric information about the direction of the temporal locking between the oscillators and depends upon the relative values of parameters of the elements. ${ }^{21}$ To visualize this effect in the network, we have simulated a network of $N=10$, fully connected Rössler oscillators and created a gray-scale, spatial map of the temporal interdependence between every element pair, as measured by the mutual values of the CEs. Black denotes $\mathrm{CE}$ values near zero, while white denotes the high-end values (typically around $S_{i j} \simeq 2$ ).

The oscillators in the array are initially ordered with respect to the value of their parameters, i.e., $a_{i}$. During the simulation the parameters of the oscillators with the lowest and highest values were switched. Figure 1 depicts the distribution of the $\mathrm{CE}$ values for the two cases (before and after the parameter change). In both cases, the CEs take the values that were analogous to the two-oscillator simulation, i.e., the oscillators with lower values of the parameter $a_{i}$ have a consistent lag with respect to the ones with higher frequencies [Fig. 1(B)]. After the parameter change [Fig. 1(C)] the values of the $\mathrm{CE}$ associated with the first oscillator or those associated with the last oscillator change rapidly, indicating that now the first oscillator is leading in the network while the last oscillator is lagging, so that the final configuration of the $S_{i j}$ again reflects the relative values of the parameter of the individual oscillators. Additionally, note that two of the oscillators have very similar values of the parameters $\left(a_{6}\right.$ $=0.271$ and $\left.a_{7}=0.272\right)$ and the values of their respective CEs converge to zero. This indicates that oscillators are completely synchronized. Figure 1(D) shows the evolution of the $\mathrm{CE}_{12}$ and $\mathrm{CE}_{21}$ as a function of time. When the oscillators' parameters are switched (dotted line) the $\mathrm{CE}$ values change rapidly and display a new state of temporal ordering in the system.

Since the measure accurately reflects the temporal relationship between pairs of oscillators in a network based on relative parameter values, it can be used to measure global ordering in a network. To this end, we define an expectivity function:

$$
E=\frac{1}{N(N-1)} \sum_{i, j, i \neq j}^{N} w_{i j},
$$

where

$$
w_{i j}=\left\{\begin{array}{cl}
1 & \text { if }\left(S_{i j}-S_{j i}\right)\left(a_{j}-a_{i}\right)>0, \\
-1 & \text { if }\left(S_{i j}-S_{j i}\right)\left(a_{j}-a_{i}\right) \leq 0 .
\end{array}\right.
$$

The expectivity function compares the measured lead/lag pattern from pairwise interactions with that which is theoretically predicted (or expected) from the relative properties of the oscillators. If the relative properties of the studied pair correctly predicts the temporal ordering within that pair (as calculated using CEs) $w_{i j}=1$, and conversely if the prediction fails $w_{i j}=-1$. Thus, $E$ links the internal properties of the coupled oscillators (relative value of parameter $a_{i}$ for the Rössler oscillators) with their relative temporal interdepen-

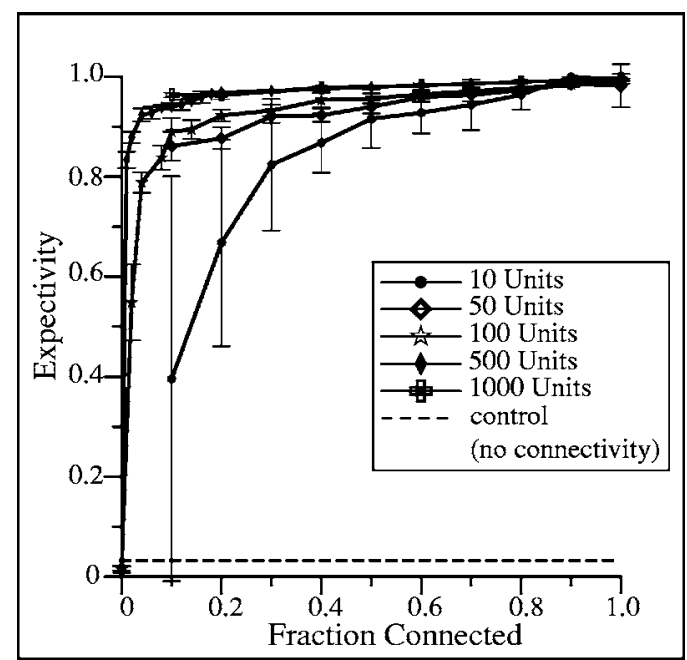

FIG. 2. Expectivity as a function of connectivity ratio for different network sizes $(N=10,50,100,500,1000)$ for random graph connectivity (except when fully connected). The expectivity reaches 1 for high connectivity ratio for all network sizes. The rate of the convergence however depends on the connectivity ratio. For a larger network, the expectivity $E \rightarrow 1$, for moderately low values of connectivity. The dotted line represents expectivity for zero connectivity (control); the coupling constant is set to $\alpha=0.4$.

dencies (lead or lag) as compared to other elements in the network. In this simulated case the comparison is obvious, as the relative properties of the coupled elements are exactly known (the values of $a_{i}$ are defined and known) -we use it to show the applicability of the expectivity metric as a global measure of temporal ordering in the system, and to infer changes in global ordering as a function of network properties. In the experimental setting the expectivity is redefined to compare an experimental observable defining the local, relative properties of the elements with the established lead/ lag pattern in the network. Thus, the expectivity in this case establishes whether the given local property of the system predicts (or is responsible for) the global ordering. ${ }^{9}$

The temporal ordering takes place even for very low connectivity $M /(N-1)$. We have calculated the expectivity for a randomly connected network (except for case of full connectivity) composed of up to 1000 Rössler oscillators (Fig. 2). Full ordering for the largest network takes place for connectivities as low as $2 \%-3 \%$. This number coincides with local connectivities observed in the brain. ${ }^{32}$

We also monitored spatio-temporal pattern formation in a one-dimensional network of Rössler oscillators $(N=100)$, having periodic boundary conditions. The system has a Small World Network topology. ${ }^{33}$ Depending on the rewiring parameter $p$, the connections in the network are local; i.e., only near neighbors are connected if $p=0$, and the network forms a random graph for $p=1$ (except the case of full connectivity). The incremental values of $p$ determine what fraction of connections is random, i.e., connecting any two elements irrespective of their distance, with respect to the local ones.

We have investigated temporal interdependencies in this network as a function of connectivity strength $(\alpha=0.4,0.5$, $0.7)$ and connectivity $[R=2,5,10$, resulting in total connectivity ratio of $M /(N-1)=2 R /(N-1)=0.04,0.10,0.20$ re- 


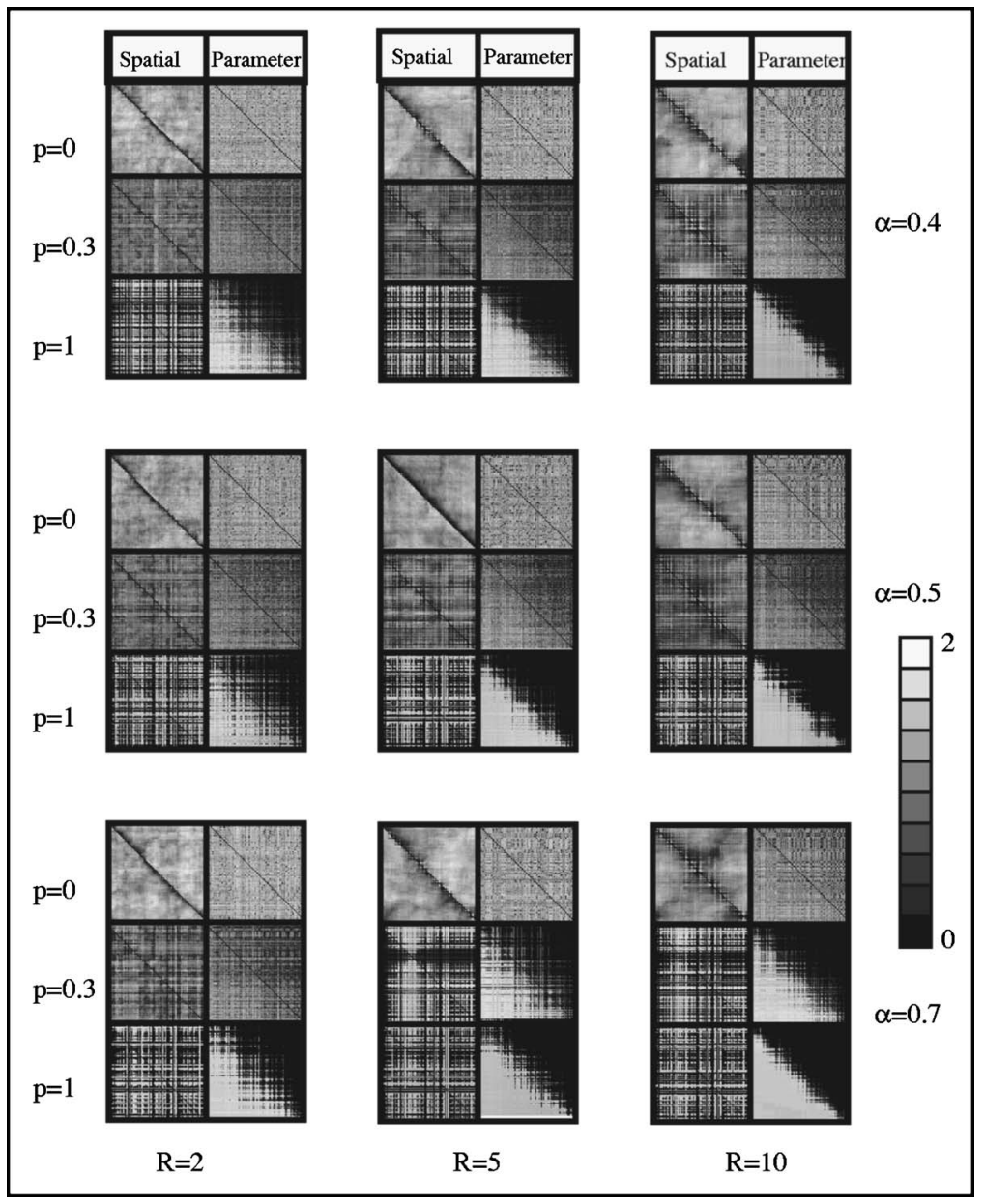

FIG. 3. Spatial maps of the CE array for a network of 100 Rössler oscillators as a function of coupling strength, connectivity radius and rewiring parameter. The leftmost columns depict array ordering by spatial relationship of oscillators within the network. The rightmost columns depict ordering by values of the oscillators' control parameter. For local connectivity, there is local, independent clustering between oscillators proximally positioned. With the increase of rewiring parameter $p$, the global order in the network is established.

spectively]. We created spatial maps of CEs that depict pattern formation in the network (Fig. 3). The maps are ordered according to relative location (spatial array) of the oscillators in the network and as a function of the values of their control parameters (parameter array). One can easily see that for all cases of local connectivity, $p=0$, the significant correlations form only near the diagonal on the spatial array, whereas there is no ordered pattern on the parameter array. The formation of small local clusters are independent of one another. However, for larger values of $p$, the ordering starts to depend on the relative values of the oscillator parameters. Near the diagonal (where the parameters are similar) small clusters of complete synchrony or even inverse lead/lag pattern can form. Those clusters form for the lowest connectivity regimes, and lowest coupling strengths, but remain locked temporally with each other. The results observed for $R=2$ and $\alpha=0.7$ confirm observations of Belykh et al. ${ }^{34}$ about formation of cluster synchrony (completely synchronized independent clusters) for local connectivity and high coupling regimes. However, for larger connectivity, the diagonal clusters start to exhibit temporal ordering (see, for example, Fig. 2, $R=5$ and $R=10, \alpha=0.7$ ).

The results depicted in the array are summarized in Figs. 4 and 5 . Figure 4 shows the expectivity as a function of the rewiring parameter $p$ for different values of coupling $\alpha$ and

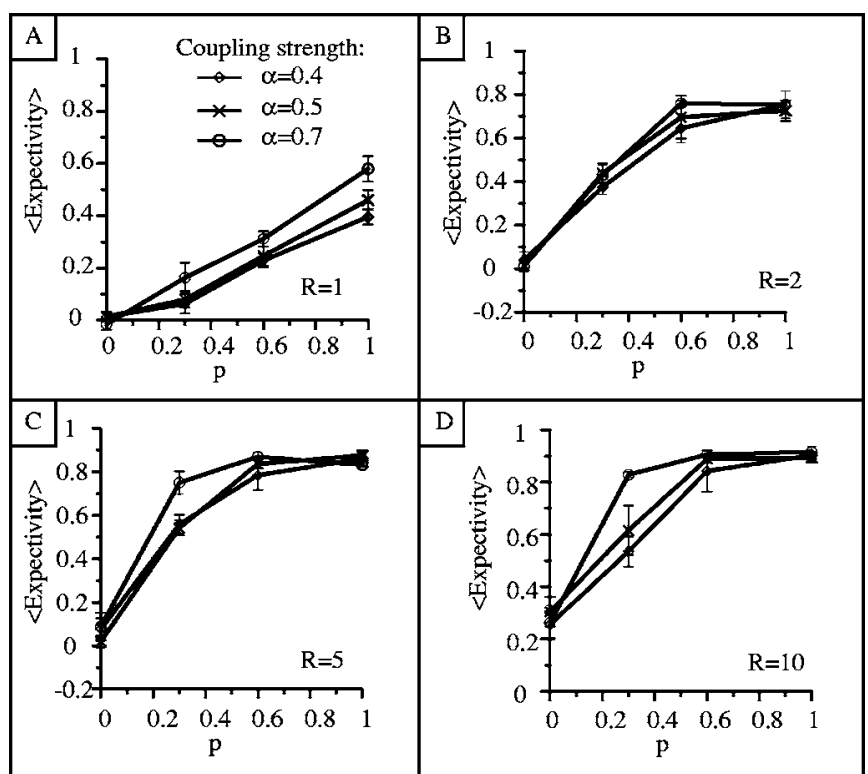

FIG. 4. Global expectivity as a function of rewiring parameter $p$ for a network of $N=100$ Rössler oscillators. The ordering in the systems increases rapidly with the changes in the network topology. For higher connectivities, the global temporal ordering is established for much lower values of rewiring parameter $p$. (A) $R=1$. (B) $R=2$. (C) $R=5$. (D) $R=10$. 


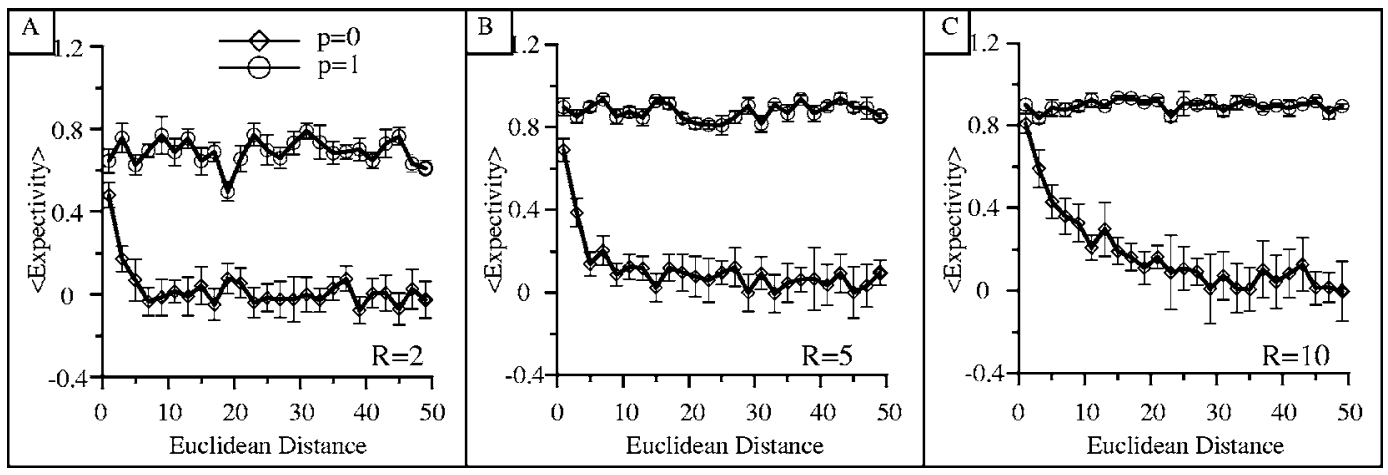

FIG. 5. Expectivity as a function of Euclidean distance between the elements in the network for a network of $N=100$ Rössler oscillators. For local connectivity $p=0$ there is temporal ordering only between proximal oscillators. For $p=1$ there is global temporal ordering between all of the oscillators in the network. The connection strength is $\alpha=0.5$. (A) $R=2$. (B) $R=5$. (C) $R=10$.

connectivity radius $R$. The global temporal ordering in the network increases significantly with the rewiring parameter $p$. The transition to global ordering strongly depends on that connectivity ratio. However, for lower connectivities the coupling strength does not influence this transition significantly. For higher connectivity ratios there are significantly faster transition for stronger coupling $(\alpha=0.7)$. Additionally, for high connectivity there is a small degree of ordering even for $p=0$ (local connectivity).

We can also use the expectivity to measure the ordering in the network as a function of the distance between the elements. Figure 5 shows values of expectivity for $p=0$ and $p=1$ as a function of Euclidean distance between any two oscillators for the three connectivity radii and $\alpha=0.5$. For $p=0$, the expectivity is relatively high for oscillators positioned near each other, and it declines exponentially as a function of distance. As expected, the decay constant for $R$ $=10$ is noticeably slower than that for $R=2$ since more oscillators are initially connected to each other. On the other hand, for $p=1$, the expectivity is much higher and independent of the distance between the oscillators.

\section{MEASUREMENT OF PATTERN FORMATION IN A BIOLOGICAL NETWORK}

In order to demonstrate the behavior of the measure in a biological application, we applied it to an in vitro biological preparation: multielectrode array recordings from cerebral ganglia of Helix snails. The procerebrum (or PC lobe) in the cerebral ganglia of slugs and snails forms a synaptically connected network and displays a spontaneous oscillatory activity in the local field potential (LFP) that is altered in frequency and amplitude by olfactory stimulation of the tentacles. ${ }^{35-37}$ The highly developed olfactory systems of slugs and snails have been used as model systems to study the neural substrates of sensory information processing and learning. ${ }^{38}$ Snails and slugs have no sense of hearing and a very primitive visual system, leaving olfaction as their most important sensory input. Olfactory stimuli are detected by sensory epithelia on the tentacles and processed in the PC lobe of the cerebral ganglia. The cerebral ganglia, the sensory epithelia and olfactory nerves connecting them can easily be isolated from the snail for study in vitro. Local field potential recordings and imaging with voltage sensitive dyes in these reduced preparations has revealed that the PC lobe displays waves of cellular excitation that propagate from one end of the PC lobe to the other. ${ }^{35,37,39}$ Olfactory stimulation of the olfactory epithelium eliminates the propagation of the wave of cellular excitation, synchronizing the oscillation across the PC lobe. ${ }^{36,39}$ This well-characterized oscillating neural network provides an interesting biological model to investigate possible existence of functional interdependencies between neurons.

The MED64 multielectrode recording system (Panasonic International Trading Corp, California) was used to record from the PC lobe of Helix aspersa, the brown garden snail. Helix snails were anesthetized by injection of $3 \mathrm{ml}$ of a solution of $50 \mathrm{mM} \mathrm{MgCl}{ }_{2}$. The central ganglia of Helix snails were dissected out of the snail preserving the connections to the olfactory nerves and olfactory epithelium on the tentacles. The cerebral ganglia were desheathed and either the right or left ganglion was placed on the MED Probe electrode bathed in a buffer containing (in $\mathrm{mM}$ ) $55.4 \mathrm{Na}^{+}$, $4.2 \mathrm{~K}^{+}, 7.0 \mathrm{Ca}^{2+}, 4.6 \mathrm{Mg}^{2+}, 80 \mathrm{Cl}^{-}, 0.2 \mathrm{H}_{2} \mathrm{PO}_{4}, 2.5 \mathrm{HCO}_{3}$, 5.0 glucose, and 10 HEPES. Extracellular recordings of the LFP oscillations in the Helix/PC lobe were made using planar, 64 electrode "Med Probes" (Alpha MED Sciences Co., LTD Japan) that consist of 64 substrate-integrated thin-film microelectrodes, of $20 \mu \mathrm{m}$ width and either 75 or $100 \mu \mathrm{m}$ between the electrodes (Fig. 6). The electrode impedance is $<22 \mathrm{k} \Omega$ at $20 \mathrm{kHz}$. The characteristics of the integrated amplifier are: Input impedance $10 \mathrm{M} \Omega$, output impedance $150 \mathrm{k} \Omega, 60 \mathrm{~dB}$ gain, and $10 \mathrm{kHz}$ bandwidth. The maximum output current is $2 \mathrm{~mA}$ and the maximum input voltage is $10 \mathrm{~V}$. The connector is used to link the probe to the amplifier through two MIO DAQ cards (National Instruments) installed in the computer running the data acquisition and analysis software. To reduce the influence of parasitic electromagnetic fields, the probe, connector, and amplifier are placed in a copper-mesh Faraday cage, the connector is grounded with a silver wire, and each electrode in grounded by one of four ground electrodes embedded in the Med Probe substrate. Spontaneous electrical activity of the Helix cerebral ganglia was recorded with a sampling rate of $20 \mathrm{kHz}$ for $50 \mathrm{~s}$. The data were preprocessed by applying 


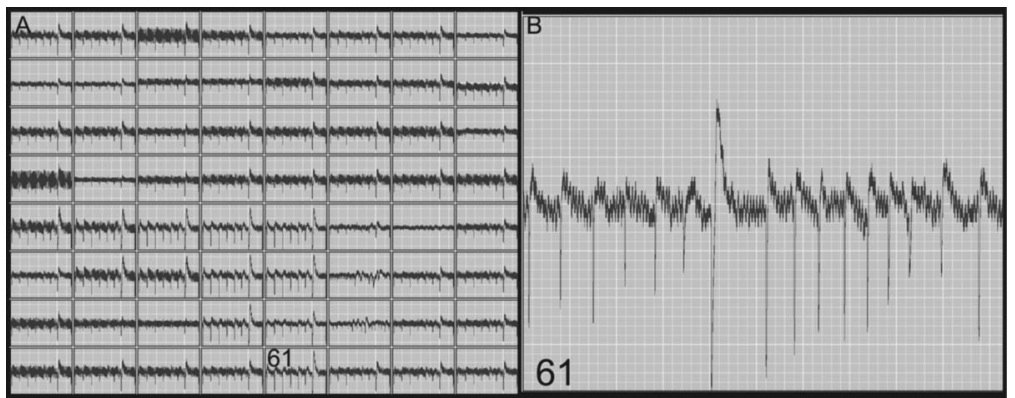

FIG. 6. Example of MED64 recording of electrical activity from the cerebral ganglion of the Helix snail. (A) Entire MED64 array. (B) An expanded view of one electrode.

infinite-impulse response (IIR) Butterworth filters to remove $60 \mathrm{~Hz}$ harmonics of power network interference (for the $k$ th harmonic, we used filters of minimal order with the stopband $k 601 \mathrm{~Hz}$ and passband starting at $k 602 \mathrm{~Hz}$. The stopband attenuation and passband ripples were 60 and $0.5 \mathrm{~dB}$, respectively). High frequency components that do not correspond to biological processes were removed using finite-impulse response low pass filter (FIR LF) with linear phase $(f s$ $=1 \mathrm{kHz}$, stopband at $1100 \mathrm{~Hz}, \quad 60 \mathrm{~dB}$ stopband attenuation). ${ }^{40}$ The dc component of each channel was removed by subtracting the average value of the channel from each sample. To efficiently assess and display oscillatory activity on 64 electrodes, spike detecting scripts to detect the peaks of the oscillation were developed using MATLAB (Mathworks; Natick, MA) and were applied to the voltage traces from the 64 electrodes. This rasterizes the data and facilitates comparison of recordings from the cerebral ganglia under different conditions.
Causal entropy differences (CEDs) were calculated for all pairs of electrodes and displayed as a function of time [Fig. 7(A)]. The event was defined as a peak of the oscillation. It can be observed that a stable temporal pattern appeared spontaneously after about $20 \mathrm{~s}$ into the recording and lasted for about $10 \mathrm{~s}$. A raster plot depicting time of population spike (dots) for every active electrode (those having more than five spikes during this epoch) was generated [Fig. 7(C)]. Average CEDs for the pairs of active electrodes were calculated and a spatial map of the $\langle\mathrm{CED}\rangle$ was created [Fig. 7(B)]. There are some electrodes (for example electrodes 31-35 and then 40-43) that exhibit a very strong and stable lead/lag pattern during this epoch, while the other electrodes seem to be largely independent. To confirm the observed pattern, we analyzed single individual bursts of activity [an example of which is depicted in Fig. 7(D)]. We observe that, as indicated by our measure, the highlighted electrode groups

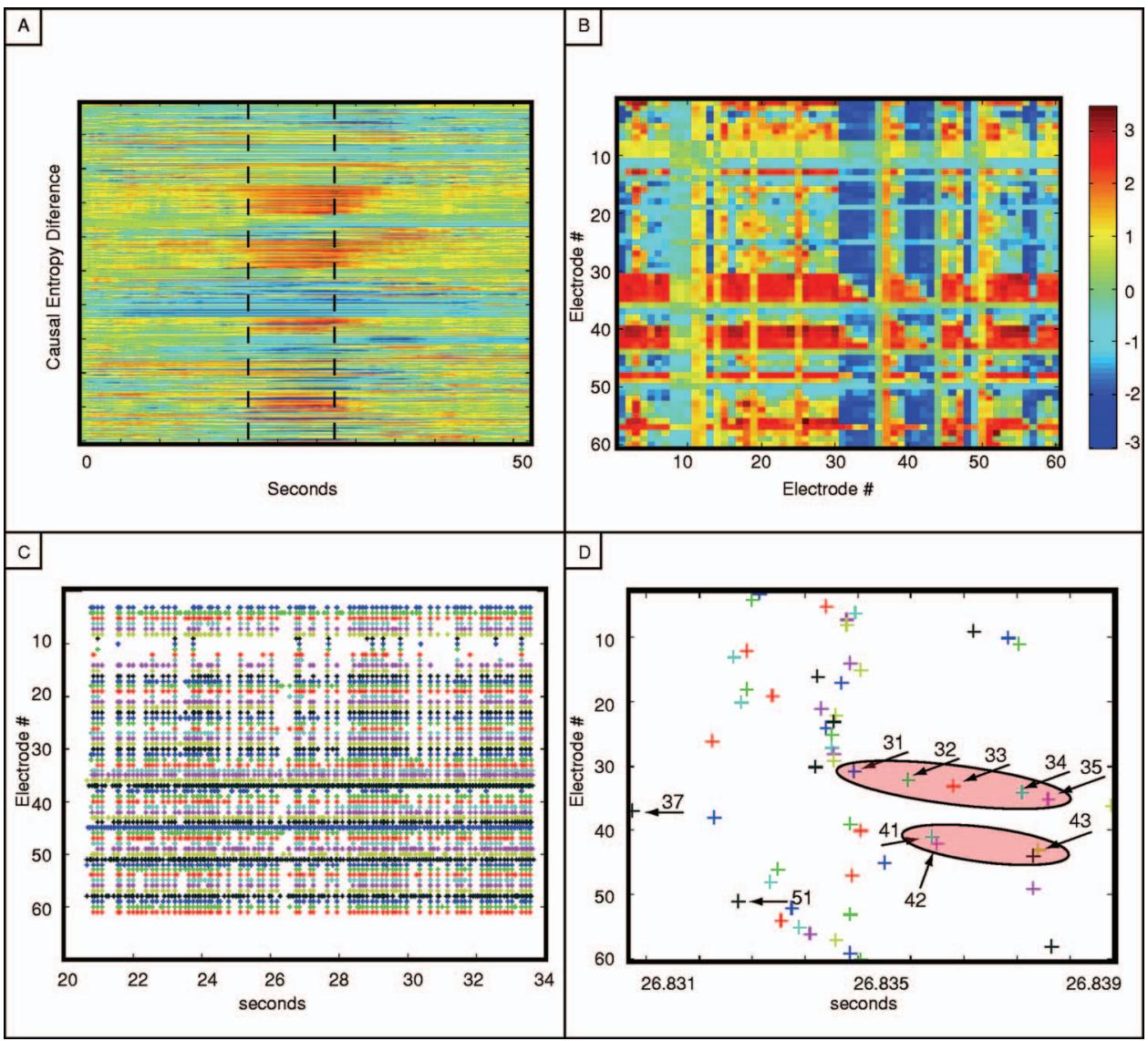

FIG. 7. (Color) Causal entropies for electrical recordings from cerebral ganglia of the Helix snail. (A) Causal entropy differences for all pairs of electrodes during the recording period. (B) Average causal entropy difference for the active electrodes during the epoch of high activity. Electrodes 31-35 and 41-43 show a strong lead/lag pattern indicating that they are summing activity from the other electrodes. (C) Raster plot of the electrodes with more than five spikes during the epoch of high activity. (D) Representative close-up of the spike times within the epoch depicted in (C). Electrodes 37 and 51 fire before the two clusters containing electrodes $31-35$ and 41-43. 
systematically activate late in the burst, whereas other electrodes (for example, electrodes 37 and 51) activate early. Note that in this particular burst there are multiple other electrodes that activate late/early. However, our CE calculations indicate that these other patterns are not stable throughout the epoch. Thus, we were able to pinpoint approximately 11 electrodes that record from populations that generate a systematic pattern of activation. This could be indicative of the fact that neurons in the vicinity of electrodes 37 and 52 tend to initiate the burst that in turn drives the electrodes 31-35 and 40-43. Note that to infer such information from visual inspection would be prohibitively difficult.

\section{DISCUSSION AND CONCLUSIONS}

We developed a CE metric that uses the relative stability of phase/lag relations to detect lead-lag patterns in the network and applied it to a system of coupled Rössler oscillators and an intact in vitro biological network to monitor the emergence of directional temporal interdependencies between elements within a network. Such temporal relationships are known to play a prominent role during information processing in neural networks and can also be used to detect a functional structure of an experimental network.

${ }^{1}$ C. Deransart, B. Hellwig, M. Heupel-Reuter, J. F. Leger, D. Heck, and C. H. Lucking, Epilepsia 44, 1513 (2003).

${ }^{2}$ R. Ferri, C. J. Stam, B. Lanuzza, F. I. Cosentino, M. Elia, S. A. Musumeci, and G. Pennisi, Clin. Neurophysiol. 115, 1202 (2004).

${ }^{3}$ W. Singer, Neuron 24, 49 (1999).

${ }^{4}$ C. M. Gray, Neuron 24, 31 (1999).

${ }^{5}$ C. von der Malsburg, Ber. Bunsenges. Phys. Chem. 89, 703 (1985).

${ }^{6}$ F. Varela, J-P. Lachaux, E. Rodriguez, and J. Martinerie, Nat. Rev. Neurosci. 2, 229 (2001).

${ }^{7}$ M. Stopfer, S. Bhagavan, B. H. Smith, and G. Laurent, Nature (London) 390, 70 (1997).

${ }^{8}$ G. Q. Bi and M. M. Poo, Nature (London) 401, 792 (1998).

${ }^{9}$ B. H. Singer, M. Derchansky, P. L. Carlen, and Michal Żochowski, Phys. Rev. E 73, 021910 (2006).

${ }^{10}$ E. N. Brown, M.Okatan, and M. A. Wilson, Neural Comput. 17, 1927 (2005).
${ }^{11}$ P. A. W. Lewis and D. R. Cox, Proceedings, Sixth Berkeley Symposium on Probability and Mathematical Statistics, 1972, Vol. 3, pp. 401-448.

${ }^{12}$ M. S. Bartlett, An Introduction to Stochastic Processes, 2nd ed. (Cambridge University Press, Cambridge, 1966).

${ }^{13}$ G. P. Moore, D. H. Perkel, and G. L. Gerstein, Biophys. J. 7, 419 (1967).

${ }^{14}$ D. H. Perkel and G. L. Gerstein, Science 164, 828 (1969).

${ }^{15}$ D. R. Brillinger, Ann. Probab. 3, 909 (1975).

${ }^{16}$ L. Lin, R. Osan, S. Shoham, W. Jin, W. Zuo, and J. Z. Tsien, Proc. Natl. Acad. Sci. U.S.A. 102, 6125 (2005).

${ }^{17}$ L. Abbott and E. Salinas, J. Comput. Neurosci. 1, 89 (1994).

${ }^{18}$ K. Zhang, I. Ginsburg, B. L. McNaughton, and T. J. Sejnowski, J. Neurophysiol. 79, 1017 (1998).

${ }^{19}$ A. F. Karr, E. S. Chornoboy, and L. P. Schramm, Biol. Cybern. 59, 265 (1988).

${ }^{20}$ I. Baruchi and E. Ben-Jacob, Neuroinformatics 2, 333 (2004).

${ }^{21}$ M. Żochowski and R. Dzakpasu, "J. Phys. A 37, 3823 (2004).

${ }^{22}$ R. Dzakpasu and M. Żochowski, Physica D 208, 115 (2005).

${ }^{23}$ M. S. Baptista, T. Pereira, J. C. Sartorelli, I. L. Caldas, and J. Kurths, Physica D 212, 216 (2005)

${ }^{24}$ M. S. Baptista and J. Kurths, Phys. Rev. E 72, 045202 (2005).

${ }^{25}$ J. Brea, D. F. Russell, and A. B. Neiman, Chaos 16, 026111 (2006).

${ }^{26}$ T. Schreiber and A. Kaiser, Int. J. Bifurcation Chaos Appl. Sci. Eng. 14, 1987 (2004).

${ }^{27}$ R. Quian Quiroga, T. Kreuz, and P. Grassberger, Phys. Rev. E 66, 041904 , 2002.

${ }^{28}$ M. G. Rosenblum, A. S. Pikovsky, and J. Kurths, Phys. Rev. Lett. 78, 4193 (1997).

${ }^{29}$ M. G. Rosenblum, A. S. Pikovsky, and J. Kurths, Phys. Rev. Lett. 76, 1804 (1996)

${ }^{30}$ D. Pazo, A. M. Zaks, and J. Kurths, Chaos 13, 309 (2003).

${ }^{31}$ G. V. Osipov, A. S. Pikovsky, M. G. Rosenblum, and J. Kurths, Phys. Rev. E 55, 2353 (1997).

${ }^{32}$ V. Braitenberg and A. Schüz, Cortex: Statistics and Geometry of Neuronal Connectivity (Springer-Verlag, Berlin, 1998).

${ }^{33}$ D. J. Watts and S. H. Strogatz, Nature (London) 393, 440 (1998).

${ }^{34}$ I. Belykh, V. Belykh, K. Nevidin, and M. Hasler, Chaos 13, 165 (2003).

${ }^{35}$ A. Gelperin and D. W. Tank, Nature (London) 345, 437 (1990).

${ }^{36}$ D. Kleinfeld, K. R. Delaney, M. S. Fee, J. A. Flores, D. W. Tank, and A. Gelperin, J. Neurophysiol. 72, 1402 (1994).

${ }^{37}$ S. Kawahara, S. Toda, Y. Suzuki, and Y. Kirino, J. Exp. Biol. 200, 1851 (1997).

${ }^{38}$ A. Gelperin, J. Exp. Biol. 202, 1855 (1999).

${ }^{39}$ K. R. Delaney, A. Gelperin, M. S. Fee, J. A. Flores, R. Gervais, D. W. Tank, and D. Kleinfeld, Proc. Natl. Acad. Sci. U.S.A. 91, 669 (1994).

${ }^{40}$ L. R. Rabiner and B. Gold, Theory and Application of Digital Signal Processing (Prentice-Hall, Englewood Cliffs, NJ, 1975). 